\title{
The Breakdown of the Minimum Polarizability Principle in Vibrational Motions as an Indicator
}

\author{
of the Most Aromatic Center
}

\author{
Miquel Torrent-Sucarrat, Josep M. Luis,* and Miquel Solà* \\ Institut de Química Computacional and Departament de Química, Universitat de Girona, E-17071 \\ Girona, Catalonia, Spain.
}

\begin{abstract}
In previous works, we have shown that bond length alternation vibrational modes in $\pi$ conjugated organic molecules may break the minimum polarizability principle (MPP). To arrive at this conclusion, we have developed a method that diagonalizes the polarizability Hessian matrix with respect to the vibrational nontotally symmetric normal coordinates. The vibrational motions that disobey the MPP in $\pi$-conjugated molecules are distortions of the equilibrium geometry that produce a reduction of the polarizability due to the localization of $\pi$-electrons. For aromatic species, this electronic localization is responsible for the subsequent reduction of the aromaticity of the system. In the present work, we apply our methodology to calculate the nontotally symmetric distortions that produce the maximum breakdown of the MPP in a series of twenty polycyclic aromatic hydrocarbons. It is shown that the nuclear displacements that break the MPP have larger components in those rings that possess the highest local aromaticity. Thus, these vibrational motions can be use as an indicator of local aromaticity.
\end{abstract}

Keywords: Minimum Polarizability Principle (MPP), conceptual Density Functional Theory (DFT), aromaticity, polarizability, vibrational modes, Maximum Hardness Principle (MHP), hardness, ab initio calculations. 


\section{INTRODUCTION}

Benzene exhibits aromaticity in all its structural and chemical manifestations. All energetic, geometric, magnetic, and reactivity based criteria of aromaticity highlight this particular property of benzene, and, consequently, this species is considered as the quintaessential and archetypical aromatic molecule. ${ }^{[1-7]}$ Among the different vibrational modes of benzene, the bond length alternation (BLA) mode of b2u symmetry (see Scheme 1), which transforms symmetric $D_{6 h}$ benzene in a Kekulé-like $D_{3 h}$ symmetry structure, has singular characteristics.

\section{SCHEME 1}

The first particular feature of this b2u vibrational mode we mention here is its surprisingly low frequency of $1309 \mathrm{~cm}^{-1}$ determined by gas phase two-photon spectroscopy measurements. ${ }^{[8]}$ Assuming that both the $\sigma$ and $\pi$-electronic systems of benzene oppose resistance to this BLA distortion ( $D_{6 h}$ to $D_{3 h}$ ), one would predict a larger frequency of about $1600 \mathrm{~cm}^{-1} \cdot{ }^{99]}$ In addition, there is a remarkable up-shift of this low frequency upon excitation to the first ${ }^{1} \mathrm{~B}_{2 \mathrm{u}}$ excited state from 1309 to $1570 \mathrm{~cm}^{-1} \cdot{ }^{[8,10,11]}$ This result, which has been confirmed theoretically through coupled cluster calculations, ${ }^{[12]}$ is totally unexpected in the context of the $\pi^{*} \leftarrow \pi$ nature of the electronic transition involved in the transit from the ${ }^{1} \mathrm{~A} 1 \mathrm{~g}$ ground state to the first ${ }^{1} \mathrm{~B}_{2 \mathrm{u}}$ excited state. Considering that the $\pi$ system is weakened in the first ${ }^{1} \mathrm{~B}_{2 u}$ excited state, one may expect a reduction in the frequency of this $b_{2 u}$ vibrational mode upon excitation. ${ }^{[13]}$ The solution to these two paradoxes came from the work of Hiberty, Shaik, and co-workers, ${ }^{[9,14-18]}$ among others, ${ }^{[19-}$ 
${ }^{21]}$ who demonstrated by means of different procedures that the $\pi$-electrons of benzene possess a distortive tendency away from the $D_{6 h}$ symmetry structure. Now it is accepted that the properties attributed to aromaticity derive from the $\pi$-delocalization, but that are the $\sigma$-electrons which are responsible for the symmetric $D_{6 h}$ framework. ${ }^{[22,23]}$ This conclusion was further substantiated by calculations of the second derivatives of orbital energies with respect to this $b_{2 u}$ normal coordinate performed by Gobbi et al. ${ }^{[24]}$ According to these authors, the second derivative of the total energy with respect to normal coordinates can be expressed in a good approximation as the sum of the second derivatives of the orbital energies, thus allowing for a $\sigma / \pi$-separation of the force constants. ${ }^{[24]}$ For the BLA $b_{2 u}$ vibration mode of benzene, it was found that the $\pi$-force constant is negative while the $\sigma$ force constant is positive. ${ }^{[24]}$ This result reinforced the conclusion that the $\pi$-system of benzene is distortive and $\pi$-delocalization is not the driving force of bond equalization in benzene.

The second particular aspect of this $b_{2 u}$ vibrational mode is that the deformation of benzene along this mode induces a partial localization of the $\pi$-electrons into localized $\pi$ bonds, reducing the aromaticity of the six-membered ring (6-MR). The effect of this distortion on the delocalization of the $\pi$-electrons in benzene was studied by Bader and co-workers ${ }^{[25]}$ considering an unsymmetrical distortion obtained by alternately increasing and decreasing the equilibrium C-C bond length of $1.42 \AA$ bond lengths to 1.54 and $1.34 \AA$, respectively. Contour maps of the Fermi-hole density indicated that there is a significant decrease in the delocalization of the $\pi$ electrons between para carbons with the distortion. ${ }^{[25]}$ Accordingly, the value of the recently defined paradelocalization index $(\mathrm{PDI})^{[26]}$ of aromaticity is reduced and this means that the movement along this vibrational mode produces a significant reduction of aromaticity. This conclusion completely agree with the harmonic oscillator model of aromaticity 


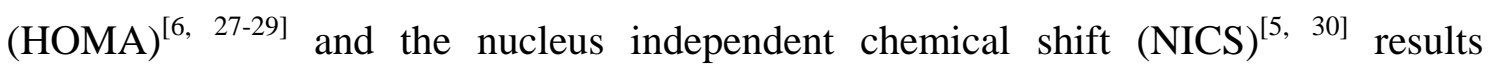
obtained by Cyrański and Krygowski, ${ }^{[31]}$ although the NICS values do not sharply differentiate between the aromaticity of benzene (-9.7 ppm) and a Kekulé structure (-8.6 ppm) with bond lengths as in ethane and ethene. ${ }^{[31]}$

Finally, another interesting property is that the $\mathrm{b}_{2 u}$ BLA vibrational mode breaks the maximum hardness (MHP) and minimum polarizability principles (MPP). ${ }^{[32]}$ These two principles together with the hard and soft acids and bases principle (HSAB) ${ }^{[33]}$ are among the most important chemical reactivity principles that have been rationalized within the framework of conceptual density functional theory (DFT). ${ }^{[34,35]}$ The MHP affirms that, at a given temperature, molecular systems evolve to a state of maximum hardness. ${ }^{[3,36-40]}$ The MPP was formulated on the basis of the MHP and an inverse relation between hardness and polarizability. ${ }^{[41]}$ This principle states that the natural direction of evolution of any system is towards a state of minimum polarizability. ${ }^{[42,43]} \mathrm{A}$ formal proof of the MHP based on statistical mechanics and the fluctuation-dissipation theorem was given by Parr and Chattaraj ${ }^{[38]}$ under the constraints that the chemical potential and the external potential must remain constant upon distortion of molecular structure. However, relaxation of these constraints seems to be permissible, and in particular, it has been found that in most cases the MHP still holds even though the chemical and external potentials vary during the molecular vibration, internal rotation or along the reaction coordinate. ${ }^{[42,44-63]}$ Hereafter, we will refer to the generalized MHP or MPP (GMHP or GMPP) as the maximum hardness or minimum polarizability principles that do not require the constancy of chemical and external potentials during molecular change.

For nontotally symmetric molecular motions and using symmetry arguments, Pearson and Palke ${ }^{[44]}$ showed that the values of the average external potential $\left(v_{e n}\right)$, hardness $(\eta)$, polarizability ( $\alpha$ ), and chemical potential ( $\mu$ ) for the positive deviation are 
the same as those for the negative deviation from equilibrium. Thus, $(\partial \eta / \partial Q)=(\partial \alpha / \partial Q)$ $=0$, and more important, $(\partial \mu / \partial Q)=\left(\partial v_{e n} / \partial Q\right)=0$ at the equilibrium geometry, where $Q$ is a nontotally symmetric normal mode coordinate. In conclusion, the chemical and external potential are roughly constant ${ }^{[32],[64]}$ for small distortions along nontotally symmetric normal modes, thus nearly following the two conditions of Parr and Chattaraj. ${ }^{[38]}$ As a consequence, the MHP and MPP are expected to be obeyed for nontotally symmetric vibrations, as confirmed by most numerical calculations of hardness and polarizability along nontotally symmetric normal modes performed so far. ${ }^{[37,44-46,65]}$ For this reason, the breakdown of the MHP and MPP for the nontotally symmetric $\mathrm{b}_{2 \mathrm{u}}$ mode of benzene is particularly relevant. In contrast, for totally symmetric distorsions, the situation is completely different. In this case, starting from the equilibrium geometry $v_{e n}$ and $\mu$ keep increasing or decreasing as the nuclei approach each other; and then, the GMPP can not be applied. Therefore, the equilibrium structure is not a maximum/minimum of hardness/polarizability for displacements along totally symmetric normal modes.

We have found in previous works ${ }^{[32,66,67]}$ small polycyclic aromatic hydrocarbons (PAHs) having nontotally symmetric BLA modes that disobey the GMHP and GMPP. The breakdown of these two principles for nontotally symmetric vibrational motions in PAHs has been extended later to molecules without $\pi$-conjugated structure or even without $\pi$ bonds. ${ }^{[68]}$ To arrive at these results, we have developed a method that diagonalizes the second derivative of the polarizability with respect to the nontotally symmetric normal coordinates $\left(\boldsymbol{\alpha}^{\prime)}\right)^{[32]}$ This method provides the distortions that produce the largest polarizability changes, which correspond to nuclear displacements that have a more marked GMPP or anti-GMPP character than the original vibrational modes.

One can expect that BLA modes in PAHs that break the GMHP and GMPP will 
have similar properties to the $\mathrm{b}_{2 \mathrm{u}}$ vibrational mode of benzene, which is, among the different vibrational modes of benzene, the one that likely produces the largest reduction of aromaticity. Indeed, the nontotally symmetric distortion that produces the maximum breakdown of the GMPP implies a distortion of the equilibrium geometry that produces the largest reduction of polarizability. In aromatic systems, this diminution of polarizability is usually related to the localization of $\pi$-electrons, which is expected to be especially important in the region with a more delocalized electron cloud. Thus, it is likely that the nontotally symmetric distortion that produces the maximum failure of the GMPP may have largest components in the most aromatic ring(s) of the system. To investigate this hypothesis, we analyze in this paper a series of PAHs having well-defined local aromaticities to determine whether nontotally symmetric vibrational distortions that disobey the MHP and MPP are mainly located in the most aromatic ring(s). Related to our study, we mention briefly that it has been found ${ }^{[69]}$ that the energy of out-of-plane deformations correlates well with changes in the degree of aromaticity of the conjugated system of whole molecules as well as specific rings. For this reason, Zhigalko and coworkers have proposed to use the frequency of the lowest out-of-plane vibration and ring deformation energy as aromaticity indexes. ${ }^{[69]}$ Finally, we must note that the GMPP is usually more restrictive that the GMHP. Then, the nontotally symmetric distortions that disobey the GMPP normally break also the GMHP (our experience indicates that a failure of the GMHP does not imply unavoidably a breakdown of the GMPP).

\section{COMPUTATIONAL DETAILS}

In this work, we have used the GAUSSIAN 98 package $^{[70]}$ to perform the geometry optimizations and polarizability, frequency, and NICS calculations. The 
diagonalization of $\alpha$ ” has been carried out at the Hartree-Fock $(\mathrm{HF})^{[71]}$ level using the Pople standard 6-31G basis set ${ }^{[72]}$ for the twenty aromatic molecules studied in this work. To analyze the basis set and electron correlation effects, HF/6-311G(d) and B3LYP/6-311G(d) ${ }^{[73-75]}$ calculations have been performed for the smallest eight PAHs analyzed. The NICS calculations have been done at the HF/6-31+G(d) level using the HF/6-31G optimized geometry.

The elements of the Hessian matrix of the polarizability with respect to the nontotally symmetric normal coordinates are calculated as

$$
\alpha_{k l} "=\left(\frac{\partial^{2} \alpha}{\partial Q_{k} \partial Q_{l}}\right)
$$

where $\alpha$ is the isotropic average polarizability

$$
\alpha=\frac{\alpha_{x x}+\alpha_{y y}+\alpha_{z z}}{3}=-\frac{1}{3}\left[\frac{\partial^{2} E}{\partial F_{x}^{2}}+\frac{\partial^{2} E}{\partial F_{y}^{2}}+\frac{\partial^{2} E}{\partial F_{z}^{2}}\right]
$$

Introducing eq 2 into 1 , one gets

$$
\alpha_{k l} "=-\frac{1}{3}\left[\frac{\partial^{4} E}{\partial Q_{k} \partial Q_{l} \partial F_{x}{ }^{2}}+\frac{\partial^{4} E}{\partial Q_{k} \partial Q_{l} \partial F_{y}{ }^{2}}+\frac{\partial^{4} E}{\partial Q_{k} \partial Q_{l} \partial F_{z}{ }^{2}}\right]=-\frac{1}{3}\left[\frac{\partial^{2} H_{k l}}{\partial F_{x}{ }^{2}}+\frac{\partial^{2} H_{k l}}{\partial F_{y}{ }^{2}}+\frac{\partial^{2} H_{k l}}{\partial F_{z}{ }^{2}}\right] \text {, }
$$

where $\mathbf{H}$ is the Hessian matrix of the energy with respect to the nontotally symmetric normal coordinates.

To evaluate $\alpha$ l” we have used two methods both of them equally correct, although they have different computational requirements. In the first method, the $\alpha_{k l}$ ” have been obtained by numerical differentiation of the analytical first derivatives of the isotropic average polarizability, $\alpha^{\prime}$. The magnitude of the displacement for the 
numerical derivatives with respect to vibrational coordinates was 0.04 a.u. This procedure requires $2 N+1$ frequency calculations, where $N$ is the number of nontotally symmetric normal coordinates. The stability of these derivatives was checked by repeating the calculation with displacements of 0.02 and 0.08 a.u. In the second method, the $\alpha_{k l}$ " elements have been calculated by second numerical differentiation of the analytical energy Hessian matrix with respect to the three directions of the static electric field $\left(F_{x}, F_{y}, F_{z}\right)$. The electric fields used for the numerical differentiation were \pm 0.0016 , $\pm 0.0032, \pm 0.0064, \pm 0.0128, \pm 0.0256$, and \pm 0.0512 a.u. (in the $x, y$, and $z$ directions). The advantage of this procedure is that it implies the same number of frequency calculations independently of the number of the molecule normal modes. We have verified further the validity of the negative signs of the eigenvalues obtained by diagonalization of the $\alpha$ " Hessian matrices by doing single-point isotropic average polarizability calculations along the postdiagonalization vibrational distortions. ${ }^{[32]}$

\section{RESULTS AND DISCUSSION}

Figure 1 depicts the molecules studied in this work, while Table 1 contains the diagonal terms and eigenvalues of $\alpha$ " corresponding to the nontotally symmetric distortions that before or/and after diagonalization break the GMPP for the eight PAHs evaluated at the HF/6-31G, HF/6-311G(d), and B3LYP/6-311G(d) levels. The same information for the rest of studied PAHs computed at the HF/6-31G level is collected in Table 2. Finally, Figure 2 depicts the postdiagonalization nuclear distortions that show the largest negative eigenvalues, which correspond to the eigenvectors with the most marked anti-GMPP character, for each aromatic system analyzed. 


\section{FIGURE 1}

In a previous study, ${ }^{[32]}$ we have established a set of simple rules to a priori predict, without calculations, whether a given $\pi$-conjugated molecule will show nontotally symmetric vibrations with an anti-GMPP character. According to these rules, nontotally symmetric vibrations in a given $\pi$-conjugated molecule will obeyed the GMPP either if the molecule does not have BLA-like vibrations or if all possible BLA movements in the molecule transform as the totally symmetric representation. On the other hand, if one or more BLA movements do not transform as the totally symmetric representation, the GMPP is disobeyed by some of the nontotally symmetric vibrational modes of the molecule.

Applying these guidelines to the twenty systems of the Figure 1, we obtain that all the molecules, except benzocyclobutadiene and benzo[g]quinoline, at least present a nontotally symmetric vibration mode that refuses to comply the GMPP. This fact is corroborated by the results collected in Tables 1 and 2. Benzocyclobutadiene and benzo[g]quinoline show special characteristics and they will be separately analyzed at the end of this section.

TABLES 1 and 2

The results of the Table 1 show that, while the diagonal elements of the $\boldsymbol{\alpha}$ ” matrix are dependent on the methodology used to compute them, the eigenvalues of the diagonalization of $\boldsymbol{\alpha}$ ” are almost totally basis set and electron correlation independent. This fact is confirmed by the displacement vectors corresponding to the postdiagonalization nuclear distortions evaluated at the HF/6-31G level and depicted in the Figure 2, which are identical to those obtained with the HF/6-311G(d) and B3LYP/6-311G(d) methods. The eigenvectors obtained from the diagonalization of $\alpha$ " indicate the linear combinations of 
nontotally symmetric vibrational modes (for a given eigenvector all implicated vibrational modes belong to the same symmetry species) that produce the largest polarizability changes.

As can be seen in Tables 1 and 2, the diagonalization of $\boldsymbol{\alpha}$ ” is required to show all the symmetry species that violate the GMPP (e.g., the b1u in anthracene or the b3g, b1u, and bзu in pentacene appear after diagonalization). The diagonalization of $\alpha$ ” also reduces the number of vibrational displacements that break the GMPP and simultaneously increases the absolute value of their negative eigenvalue. This concentration of information facilitates the analysis of our results. In the case of the acenaphtylene at the B3LYP/6-311G(d) level, the diagonalization of $\boldsymbol{\alpha}$ ” is essential to find molecular distortions that do not follow the GMPP, displaying the utility of this method to detect this kind of molecular distortions.

\section{FIGURE 2}

We shall begin our discussion on the relation between the breakdown of GMPP and aromaticity by analyzing first the two molecules (benzene and naphthalene) that have a unique type of ring. In these two systems, the postdiagonalization nuclear distortions of the C atoms display an evident BLA distortion (see Figure 2). On the other hand, biphenylene, acenaphthylene, fluorene, and anthracene-9,10-dione show two types of rings - one is an aromatic 6-MR (rings A of the Figure 1) with a negative value of NICS and the other is a non-aromatic center (rings B) with a positive value of NICS. The differentiation between the two rings is also reflected in the Figure 2, where only the most aromatic ring A presents the expected BLA distortion. Pyracylene, a controversial aromatic system, ${ }^{[26,76,77]}$ presents a similar structure with two different rings. It contains a 5-MR (center B) that is clearly non-aromatic according to magnetic (ring currents and NICS), ${ }^{[77]}$ geometric (HOMA), ${ }^{[26]}$ 
and electronic (aromatic fluctuation index, FLU) criteria of aromaticity. ${ }^{[76]}$ Moreover it presents a 6-MR (center A) with an intermediate aromaticity as indicated by HOMA, ring currents, PDI, and FLU results. ${ }^{[76,77]}$ However, according to NICS values this 6-MR possesses only a slight aromaticity. A recent work ${ }^{[77]}$ has shown that the magnetic field produced by paratropic ring currents of the adjacent 5-MRs in pyracylene leads to an artificial underestimation of the aromaticity of the 6-MRs by NICS. This result supports the intermediate aromatic character of the 6-MRs in pyracylene. Accordingly, the postdiagonalization distortion depicted in Figure 2 presents a negative eigenvalue with a clear BLA movement in the aromatic 6-MRs (note that one can not construct a BLA movement in an odd-membered ring).

The anthracene, acridine, phenanthrene, and tetracene molecules present two types of aromatic 6-MRs with negative values of NICS. As can be seen in Figure 2, the BLA postdiagonalization distortions properly indicate the most aromatic center of these molecules. In a similar way, the [4]helicene, triphenylene, and chrysene contain two types of aromatic 6-MRs. For these molecules, the postdiagonalization displacements are less informative than in the previous systems since all the $\mathrm{C}$ atoms show important translations. However, only the most aromatic 6-MR presents a clear BLA, i.e., an alternation of increased and decreased adjacent bond lengths around the 6-MR.

It is worth nothing that perylene is the only studied molecule with two large negative eigenvalues (-3.273 and -3.106 with b3g and b2u symmetry, respectively). As can be seen in the Figure 2, the postdiagonalization distortion of b3g symmetry shows a quasiBLA movement for ring A (see Figure 1), while the center B presents a sequence of shortlong-long-short-long distances, indicating that the most aromatic center is ring A. In contrast, the $\mathrm{b}_{2 \mathrm{u}}$ distortion displays a BLA in the six rings, without making an obvious differentiation between centers A and B. 
Finally, the [5]helicene, pentacene, and picene contain three kinds of aromatic 6MRs with negative values of NICS. The method of diagonalization of $\alpha$ ” in the pentacene molecule allows by only looking at the b2u distortion of Figure 2 to determine the relative aromaticity order of the three rings. While ring A contains fix atoms, rings B and C show significant displacements of the carbon atoms, although only the most aromatic ring $\mathrm{C}$ displays a BLA distortion. This result is consistent with the NICS values indicating that ring $\mathrm{A}$ is more aromatic than $\mathrm{B}$, and this, in turn, more aromatic than $\mathrm{C}$. On the other hand, the postdiagonalization displacements in [5]helicene and picene simply show the largest components of the BLA mode in the most aromatic ring.

At the beginning of this section, we mention that the benzocyclobutadiene and benzo[g]quinoline show special characteristics. The diagonalization of $\boldsymbol{\alpha}$ ” with respect to the nontotally symmetric normal coordinates and the direct application of the set of simple rules based on the symmetry of the possible BLA movements lead to the same conclusion, that is, the GMPP is obeyed by all nontotally symmetric distortions of these molecules. Thus, with the present methodology is not possible to ascertain the most aromatic center in these molecules. As an alternative way, we have investigated whether the diagonalization of $\alpha$ " with respect to the totally symmetric normal coordinates can provide information that can not be obtained in this case from the nontotally symmetric vibrations. It is important to remark that totally symmetric distortions at the equilibrium geometry are neither a maximum nor minimum of properties such as $\alpha, \mu$, or $v_{e n}$. Notwithstanding, their eigenvalues apprise the curvature of the polarizability along these symmetric displacements and indicate whether we are near or far from a polarizability maximum or minimum.

As can be seen in the Figure 2, the application of this method to the benzocyclobutadiene and benzo[g]quinoline helps to determine the relative aromatic character of the different rings. In benzo[g]quinoline, this method only points out the most 
aromatic ring; nevertheless, the difference of aromaticity between centers $\mathrm{A}$ and $\mathrm{C}$ is small. Certainly, it is possible to apply the diagonalization of the polarizability Hessian along totally symmetric modes to the rest of the molecules, although the results do not provide much more information than that obtained from the diagonalization along nontotally symmetric modes. For instance, using this methodology to systems like naphthalene, biphenylene, or fluorene one obtains large negative eigenvalues with postdiagonalized totally symmetric movements, which give exactly the same information than the diagonalization with respect to the nontotally symmetric displacements. In contrast, systems like benzene, pyracylene, or perylene only show positive eigenvalues or few small negative eigenvalues that have postdiagonalization distortions without any relation with BLA motions and aromaticity. Nevertheless, in some cases the diagonalization along totally symmetric modes can be useful to complement the information obtained from the nontotally symmetric modes. As an example, the totally symmetric postdiagonalization movements of [5]helicene and picene (see Figure 3) differentiate the two most aromatic rings (centers A and C), which show BLA distortion from the less aromatic ring (center B). However, at variance with the nontotally symmetric, totally symmetric postdiagonalization displacements assign similar aromatic character to centers A and C.

FIGURE 3

\section{CONCLUSIONS}

The aromaticity is a property associated with the cyclic delocalization of $\pi$ electrons, resulting in an extra stabilization of aromatic molecules and a desestabilization in antiaromatic species. The evaluation of aromaticity is usually based on the classical aromaticity criteria: structural, magnetic, energetic, and reactivity-based measures. In this 
work, we have introduced a new way to qualitatively determine the most aromatic region of a PAH. This method finds the nontotally symmetric BLA distortions that produce the maximum nonfulfillment of the GMPP by means of the diagonalization of the polarizability Hessian matrix with respect to the vibrational nontotally symmetric normal coordinates. Then, our new method assigns the highest local aromaticity to the rings that have the largest components of such anti-GMPP vibrational displacements. We have applied this methodology to a large set of PAHs and we have shown that predictions of the most aromatic centers given by the present method and those expected from NICS calculations fully coincide.

Acknowledgements. Support for this work from the Spanish Ministerio de Ciencia y Tecnología (projects No. BQU2002-04112-C02-02 and BQU2002-03334), from the DURSI (Generalitat de Catalunya) (project 2001SGR-00290), and the use of the computational facilities of the Catalonia Supercomputer Center (CESCA) are gratefully acknowledged. M. T.-S. is financed through CIRIT Project No. FI/01-000699 from the Generalitat de Catalunya. M. S. thanks the DURSI for financial support through the Distinguished University Research Promotion, 2001. 


\section{References and notes}

[1] P. J. Garrat, Aromaticity, John Wiley \& Sons, New York, 1986.

[2] V. I. Minkin, M. N. Glukhovtsev and B. Y. Simkin, Aromaticity and Antiaromaticity: Electronic and Structural Aspects, John Wiley \& Sons, New York, 1994.

[3] F. De Proft and P. Geerlings, Chem. Rev. 2001, 101, 1451-1464.

[4] D. Lloyd, J. Chem. Inf. Comp. Sci. 1996, 36, 442-447.

[5] P. v. R. Schleyer and H. Jiao, Pure Appl. Chem. 1996, 68, 209-218.

[6] T. M. Krygowski, M. K. Cyrañski, Z. Czarnocki, G. Häfelinger and A. R. Katritzky, Tetrahedron 2000, 56, 1783-1796.

[7] P. v. R. Schleyer, Chem. Rev. 2001, 101, 1115-1118.

[8] L. Wunsch, H. J. Neusser and E. W. Schlag, Chem. Phys. Lett. 1975, 31, 433-440.

[9] P. C. Hiberty and S. S. Shaik, Phys. Chem. Chem. Phys. 2004, 6, 224-231.

[10] N. Mikami and M. Ito, J. Chem. Phys. 1976, 64, 3077-3078.

[11] L. Wunsch, F. Metz, H. J. Neusser and E. W. Schlag, J. Chem. Phys. 1977, 66, 386-400.

[12] O. Christiansen, J. F. Stanton and J. Gauss, J. Chem. Phys. 1998, 108, 3987-4001.

[13] M. J. Robey and E. W. Schlag, J. Chem. Phys. 1977, 67, 2775-2781.

[14] S. S. Shaik and P. C. Hiberty, J. Am. Chem. Soc. 1985, 107, 3089-3095.

[15] S. S. Shaik, P. C. Hiberty, J.-M. Le Four and G. Ohanessian, J. Am. Chem. Soc. 1987, 109, 363-374.

[16] P. C. Hiberty, D. Danovich, A. Shurki and S. Shaik, J. Am. Chem. Soc. 1995, 117, 7760-7768.

[17] K. Jug, P. C. Hiberty and S. S. Shaik, Chem. Rev. 2001, 101, 1477-1500.

[18] S. S. Shaik, A. Shurki, D. Danovich and P. C. Hiberty, Chem. Rev. 2001, 101, 1501-1539.

[19] R. S. Berry, J. Chem. Phys. 1961, 35, 2253-2254.

[20] A. Stanger and E. Tkachenko, J. Comput. Chem. 2001, 22, 1377-1386.

[21] B. Kovacevic, D. Baric, Z. B. Maksic and T. Müller, ChemPhysChem 2004, 5, $1352-1364$

[22] G. P. Bean, J. Org. Chem. 1998, 63, 2497-2506.

[23] N. Sadlej-Sosnowska, J. Org. Chem. 2001, 66, 8737-8743. 
[24] A. Gobbi, Y. Yamaguchi, G. Frenking and H. F. Shaefer III, Chem. Phys. Lett. 1995, 244, 27-31.

[25] R. F. W. Bader, A. Streitwieser, A. Neuhaus, K. E. Laidig and P. Speers, J. Am. Chem. Soc. 1996, 118, 4959-4965.

[26] J. Poater, X. Fradera, M. Duran and M. Solà, Chem. Eur. J. 2003, 9, 400-406.

[27] J. Kruszewski and T. M. Krygowski, Tetrahedron Lett. 1972, 13, 3839-3842.

[28] T. M. Krygowski and M. K. Cyrañski, Chem. Rev. 2001, 101, 1385-1419.

[29] T. M. Krygowski, J. Chem. Inf. Comp. Sci. 1993, 33, 70-78.

[30] P. v. R. Schleyer, C. Maerker, A. Dransfeld, H. Jiao and N. J. R. van Eikema Hommes, J. Am. Chem. Soc. 1996, 118, 6317-6318.

[31] M. K. Cyrañski and T. M. Krygowski, Tetrahedron 1999, 55, 6205-6210.

[32] M. Torrent-Sucarrat, J. M. Luis, M. Duran and M. Solà, J. Chem. Phys. 2002, 117, 10651-10570.

[33] R. G. Pearson, Chemical Hardness: Applications from Molecules to Solids, WileyVCH, Oxford, 1997.

[34] P. Geerlings, F. De Proft and W. Langenaeker, Chem. Rev. (Washington, D. C.) 2003, 103, 1793-1873.

[35] H. Chermette, J. Comput. Chem. 1999, 20, 129-154.

[36] R. G. Pearson, J. Chem. Educ. 1987, 64, 561-567.

[37] R. G. Pearson, J. Chem. Educ. 1999, 76, 267-275.

[38] R. G. Parr and P. K. Chattaraj, J. Am. Chem. Soc. 1991, 113, 1854-1855.

[39] R. G. Pearson in Chemical Hardness - A historical Introduction, Vol. 80 SpringerVerlag, Berlin, 1993, pp. 1-10.

[40] P. K. Chattaraj and R. G. Parr in Density functional theory of chemical hardness, Vol. 80 Springer-Verlag, Berlin, 1993, pp. 11-25.

[41] T. K. Ghanty and S. K. Ghosh, J. Phys. Chem. 1993, 97, 4951-4953.

[42] T. K. Ghanty and S. K. Ghosh, J. Phys. Chem. 1996, 100, 12295-12298.

[43] P. K. Chattaraj and S. Sengupta, J. Phys. Chem. 1996, 100, 16126-16130.

[44] R. G. Pearson and W. E. Palke, J. Phys. Chem. 1992, 96, 3283-3285.

[45] S. Pal, N. Naval and R. Roy, J. Phys. Chem. 1993, 97, 4404-4406.

[46] G. Makov, J. Phys. Chem. 1995, 99, 9337-9339.

[47] G. I. Cárdenas-Jirón, J. Lahsen and A. Toro-Labbé, J. Phys. Chem. 1995, 99, 53255330 . 
[48] G. I. Cárdenas-Jirón and A. Toro-Labbé, J. Phys. Chem. 1995, 99, 12730-12738.

[49] G. I. Cárdenas-Jirón, S. Gutiérrez-Oliva, J. Melin and A. Toro-Labbé, J. Phys. Chem. A 1997, 101, 4621-4627.

[50] G. I. Cárdenas-Jirón and A. Toro-Labbé, J. Mol. Struct. (Theochem) 1997, 390, 7989.

[51] S. Gutiérrez-Oliva, J. R. Letelier and A. Toro-Labbé, Mol. Phys. 1999, 96, 61-70.

[52] P. K. Chattaraj and A. Poddar, J. Phys. Chem. A 1999, 103, 1274-1275.

[53] Z. Zhou and R. G. Parr, J. Am. Chem. Soc. 1989, 111, 7371-7379.

[54] D. Datta, J. Phys. Chem. 1992, 96, 2409-2410.

[55] T. Kar and S. Scheiner, J. Phys. Chem. 1995, 99, 8121-8124.

[56] P. K. Chattaraj, A. Cedillo, R. G. Parr and E. M. Arnett, J. Org. Chem. 1995, 60, 4707-4714.

[57] T. Kar, S. Scheiner and A. B. Sannigrahi, J. Phys. Chem. A 1998, 102, 5967-5973.

[58] T. Mineva, E. Sicilia and N. Russo, J. Am. Chem. Soc. 1998, 120, 9053-9058.

[59] A. Toro-Labbé, J. Phys. Chem. A 1999, 103, 4398-4403.

[60] M. Solà and A. Toro-Labbé, J. Phys. Chem. A 1999, 103, 8847-8852.

[61] P. K. Chattaraj, P. Fuentealba, B. Gómez and R. Contreras, J. Am. Chem. Soc. 2000, 122, 348-351.

[62] U. Hohm, J. Phys. Chem. A 2000, 104, 8418-8423.

[63] E. Sicilia, N. Russo and T. Mineva, J. Phys. Chem. A 2001, 105, 442-450.

[64] By definition $v_{\mathrm{en}}=\int \rho(\vec{r}) v(\vec{r}) d \vec{r}$, and therefore if $v_{\mathrm{en}}$ keeps constant for a particular distortion of the molecular structure, one may assume that $v(\vec{r})$ also remains roughly constant during this distortion.

[65] P. K. Chattaraj, P. Fuentealba, P. Jaque and A. Toro-Labbé, J. Phys. Chem. A 1999, 103, 9307-9312.

[66] M. Torrent-Sucarrat, J. M. Luis, M. Duran and M. Solà, J. Am. Chem. Soc. 2001, 123, 7951-7952.

[67] L. Blancafort, M. Torrent-Sucarrat, J. M. Luis, M. Duran and M. Solà, J. Phys. Chem. A 2003, 38, 7337-7339.

[68] M. Torrent-Sucarrat, M. Duran, J. M. Luis and M. Solà, J. Phys. Chem. A 2005, 109, 615-621.

[69] M. V. Zhigalko, O. V. Shishkin, L. Gorb and J. Leszczynski, J. Mol. Struct. (Theochem) 2004, 693, 153-159. 
[70] M. J. Frisch, G. W. Trucks, H. B. Schlegel, G. E. Scuseria, M. A. Robb, J. R. Cheeseman, V. G. Zakrzewski, J. A. Montgomery, R. E. Stratmann, J. C. Burant, S. Dapprich, J. M. Millam, A. D. Daniels, K. N. Kudin, M. C. Strain, O. Farkas, J. Tomasi, V. Barone, M. Cossi, R. Cammi, B. Mennucci, C. Pomelli, C. Adamo, S. Clifford, J. Ochterski, G. A. Petersson, P. Y. Ayala, Q. Cui, K. Morokuma, P. Salvador, J. J. Dannenberg, D. K. Malick, A. D. Rabuck, K. Raghavachari, J. B. Foresman, J. Cioslowski, J. V. Ortiz, A. G. Baboul, B. B. Stefanov, G. Liu, A. Liashenko, P. Piskorz, I. Komaromi, R. Gomperts, R. L. Martin, D. J. Fox, T. Keith, M. Al-Laham, C. Peng, A. Nanayakkara, M. Challacombe, P. M. W. Gill, B. G. Johnson, W. Chen, M. W. Wong, J. L. Andres, R. Gonzalez, M. Head-Gordon, E. S. Replogle and J. A. Pople in Gaussian 98, Vol. Gaussian 98, rev. A11, Pittsburgh, PA, 1998.

[71] C. C. J. Roothaan, Rev. Mod. Phys. 1951, 23, 69.

[72] W. J. Hehre, L. Radom, P. v. R. Schleyer and J. A. Pople in Vol. Wiley, New York, 1986.

[73] A. D. Becke, J. Chem. Phys. 1993, 98, 5648-5652.

[74] C. Lee, W. Yang and R. G. Parr, Phys. Rev. B 1988, 37, 785-789.

[75] P. J. Stephens, F. J. Devlin, C. F. Chabalowski and M. J. Frisch, J. Phys. Chem. 1994, 98, 11623-11627.

[76] E. Matito, M. Duran and M. Solà, J. Chem. Phys. 2005, 122.

[77] J. Poater, M. Solà, R. G. Viglione and R. Zanasi, J. Org. Chem. 2004, 69, 75377542. 


\section{Scheme and Figure Captions}

Scheme 1: Transformation of the $D_{6 h}$ benzene in a Kekulé-like $D_{3 h}$ symmetry structure due to the bond length alternation (BLA) mode of b2u symmetry.

Figure 1: A schematic representation of the molecules studied in this work.

Figure 2: Schematic representation of the displacement vectors corresponding to the postdiagonalization nuclear distortions that show the most marked anti-GMPP character of each aromatic molecule (the most negative eigenvalue) obtained at the HF/6-31G level. The depicted displacement vectors of the non-hydrogen atoms have been multiplied by two in the representation for seek of clarity. The numbers inside the molecular rings are the NICS values of these rings calculated at the HF/6-31+G(d) level with the HF/6-31G optimized geometry.

Figure 3: Schematic representation of the displacement vectors corresponding to the totally symmetric postdiagonalization nuclear distortions that show the two most negative eigenvalues of [5]helicene and picene obtained at the HF/6-31G level. The depicted displacement vectors of the non-hydrogen atoms have been multiplied by two in the representation for seek of clarity. The numbers inside the molecular rings are the NICS values of these rings calculated at the HF/6-31+G(d) level with the HF/6-31G optimized geometry. The numbers between brackets are the eigenvalues of the postdiagonalization nuclear distortions. 


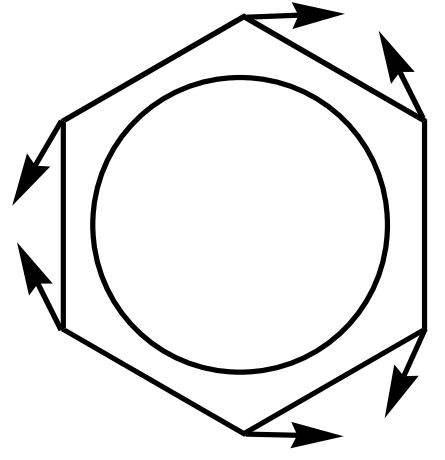

$D_{6 h}$

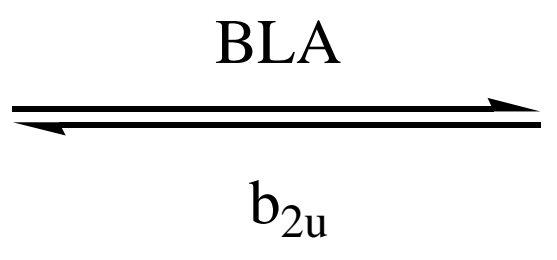

Scheme 1

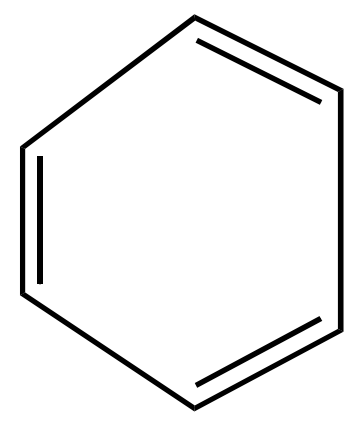

$D_{3 h}$ 
1<smiles>C1=Cc2ccccc21</smiles><smiles>c1ccc2ccccc2c1</smiles>

Naphthalene $D_{2 h}$<smiles>c1ccc2c(c1)-c1ccccc1-2</smiles>

Biphenylene $D_{2 h}$

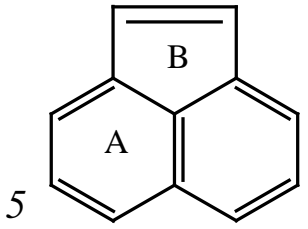

Acenaphthylene $\mathrm{C}_{2 v}$

Benzene $D_{6 h} \quad$ Benzocyclobutadiene $\mathrm{C}_{2 v}$<smiles>C1=Cc2ccc3c4c(ccc1c24)C=C3</smiles>

Pyracylene $D_{2 h}$

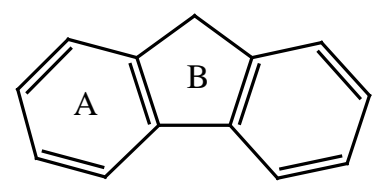

7<smiles></smiles>

Anthracene $D_{2 h}$<smiles>O=C1c2ccccc2C(=O)c2ccccc21</smiles>

Anthracene-9,10-dione $D_{2 h}$<smiles>c1ccc2nc3ccccc3cc2c1</smiles>

Acridine $C_{2 v}$<smiles>c1ccc2c(c1)ccc1ccc3ccccc3c12</smiles>

[4]helicene $C_{2}$

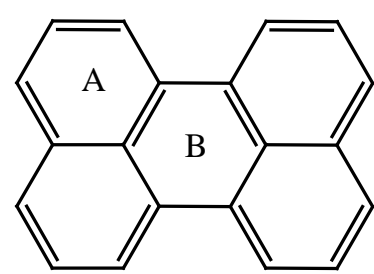<smiles>c1ccc2cc3ncccc3cc2c1</smiles>

Benzo $[g]$ quinoline $C_{s}$<smiles>c1ccc2cc3cc4ccccc4cc3cc2c1</smiles>

14

Tetracene $D_{2 h}$<smiles>c1ccc2c(c1)c1ccccc1c1ccccc21</smiles>

Triphenylene $D_{3 h}$
12

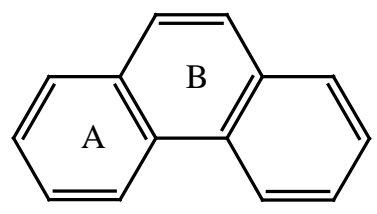

Phenanthrene $C_{2 v}$
Perylene $D_{2 h}$

16<smiles>c1ccc2cc3cc4cc5ccccc5cc4cc3cc2c1</smiles>

20

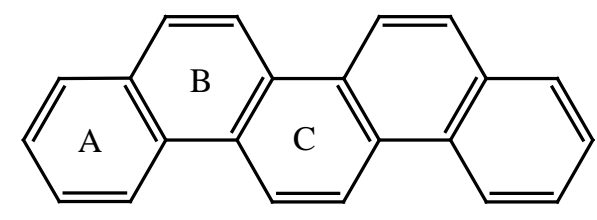

Picene $C_{2 v}$ 

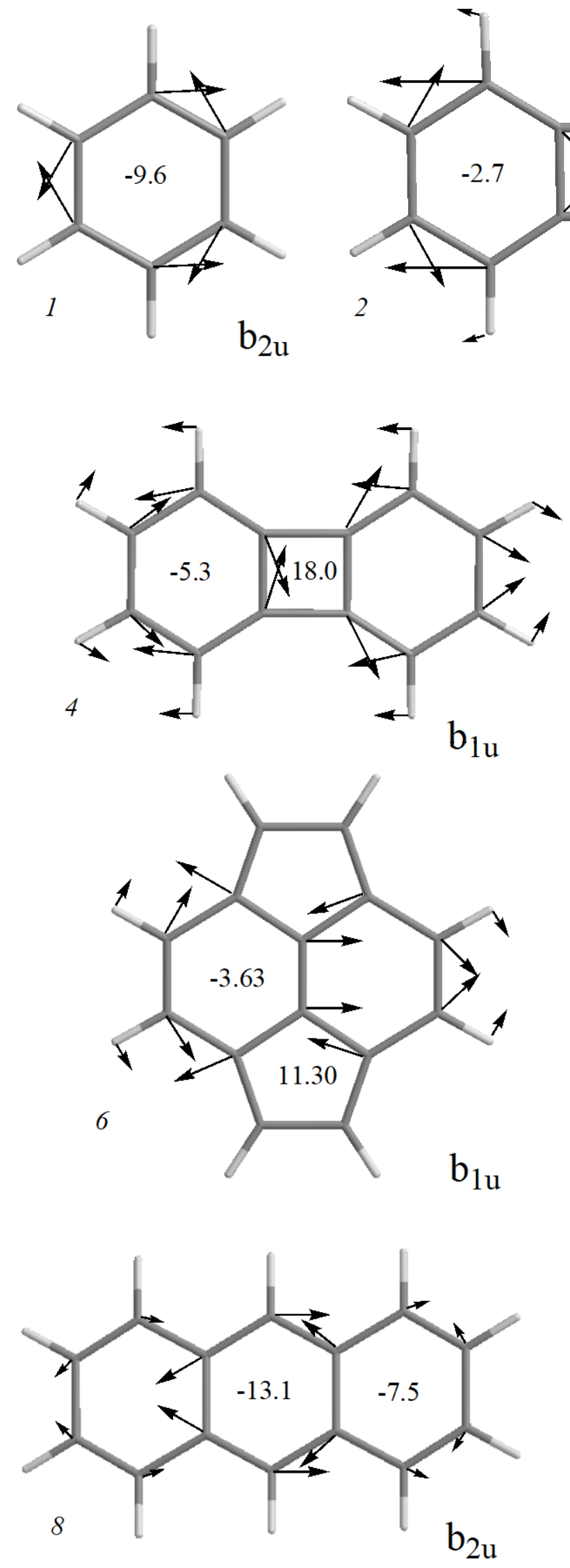

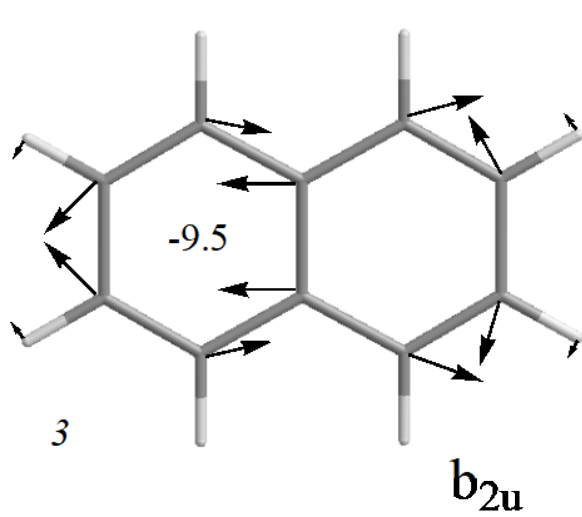

5
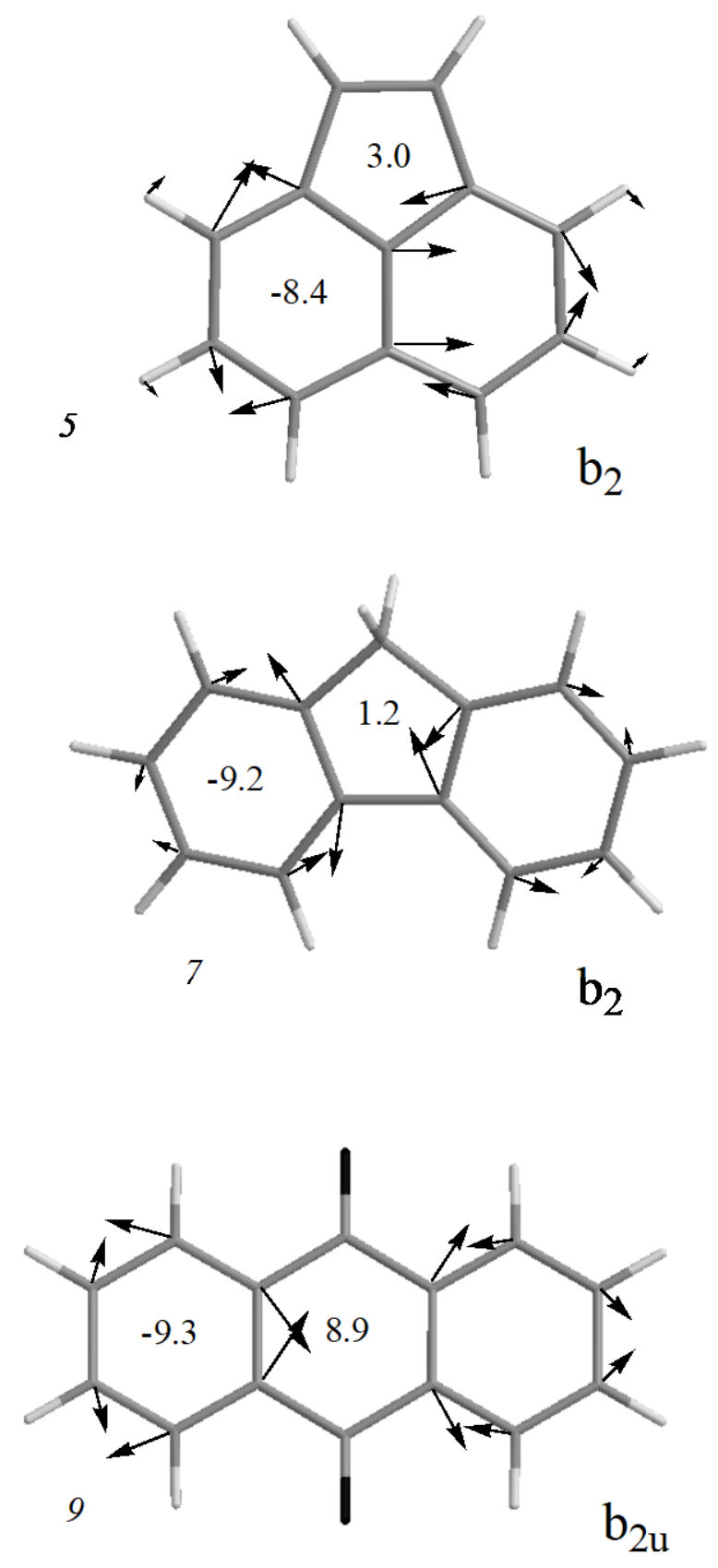

Figure 2a 

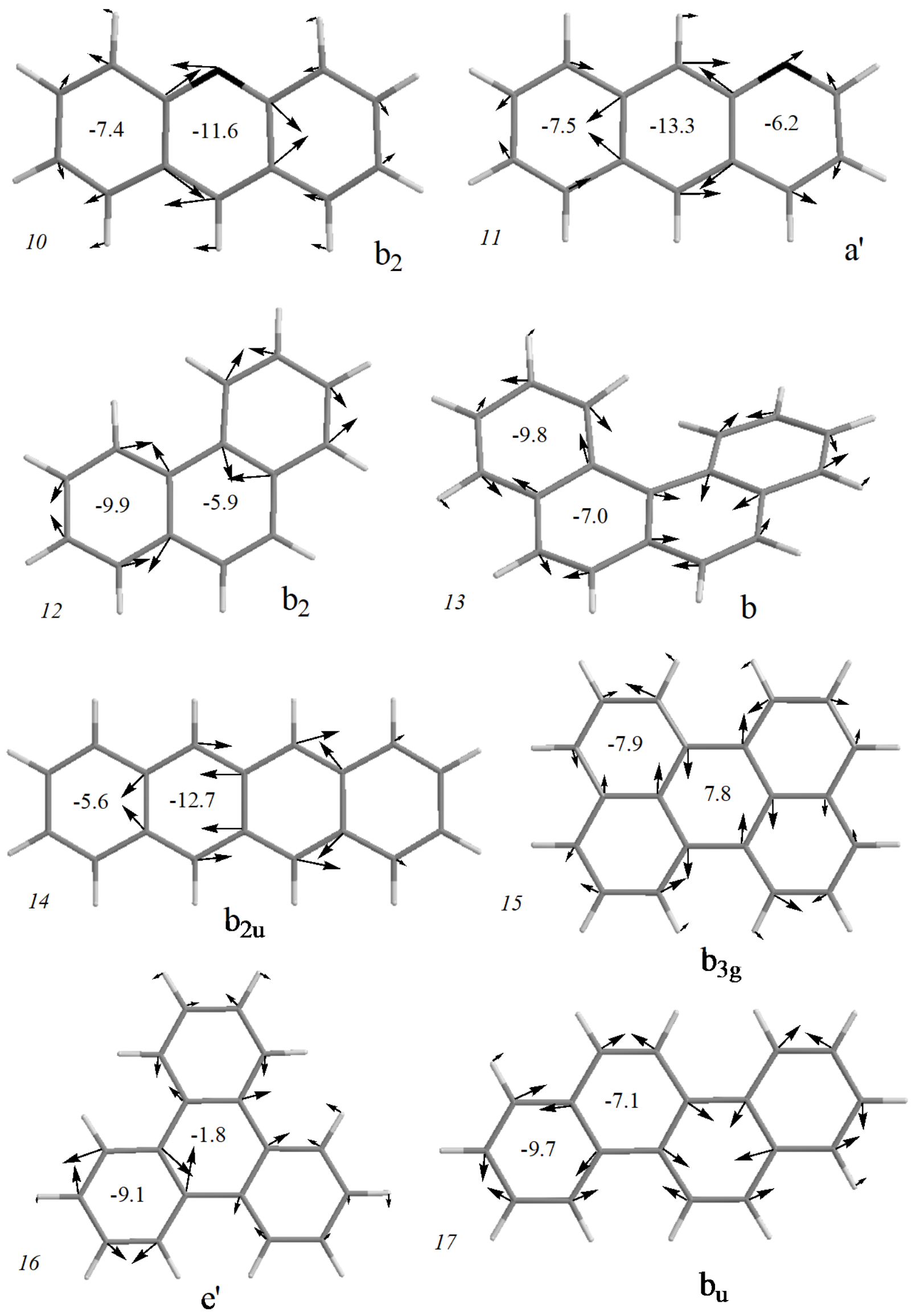

Figure 2b 


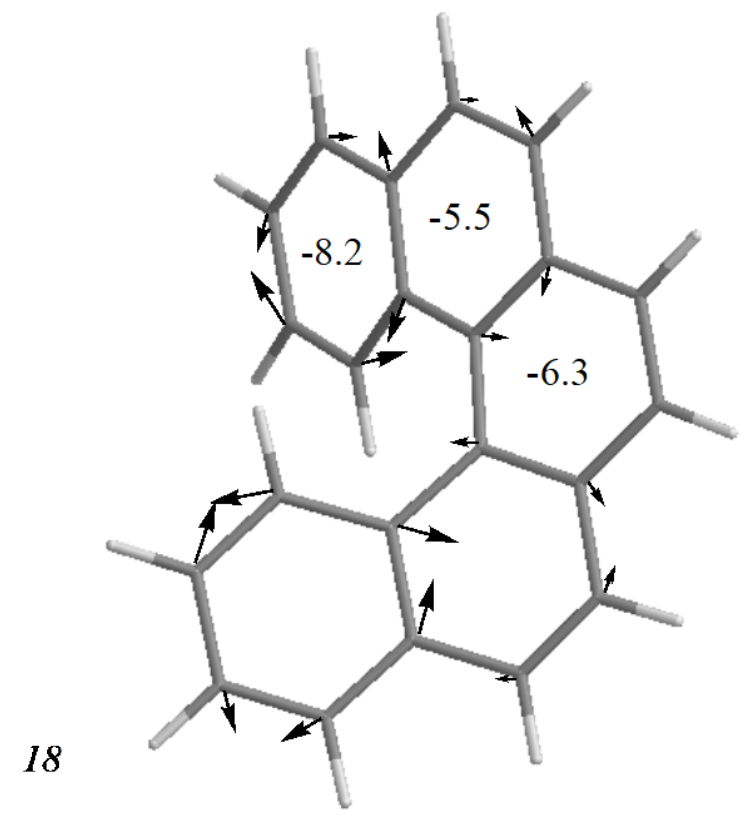

b

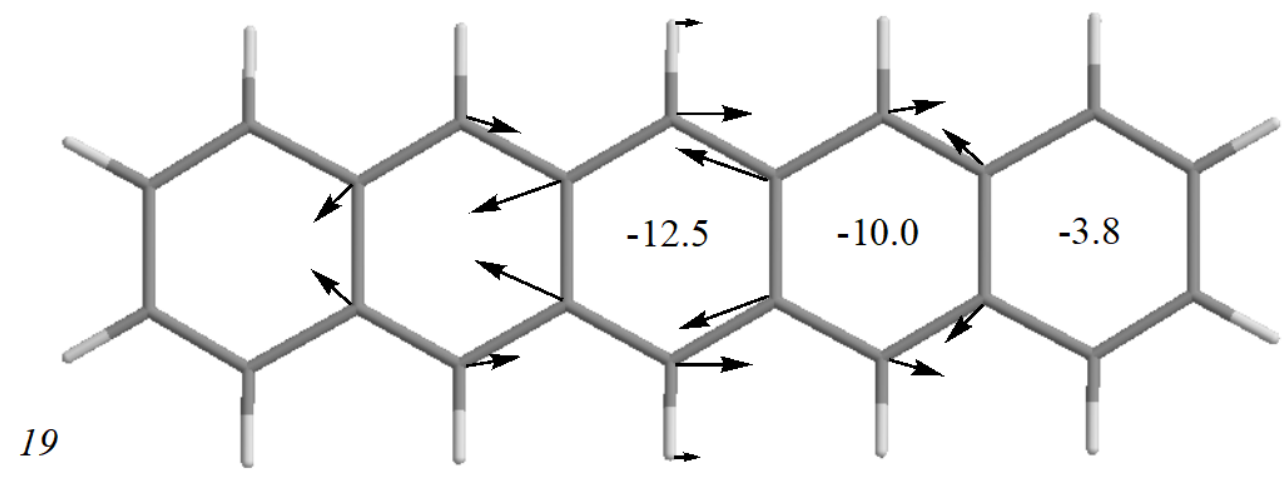

$b_{2 u}$

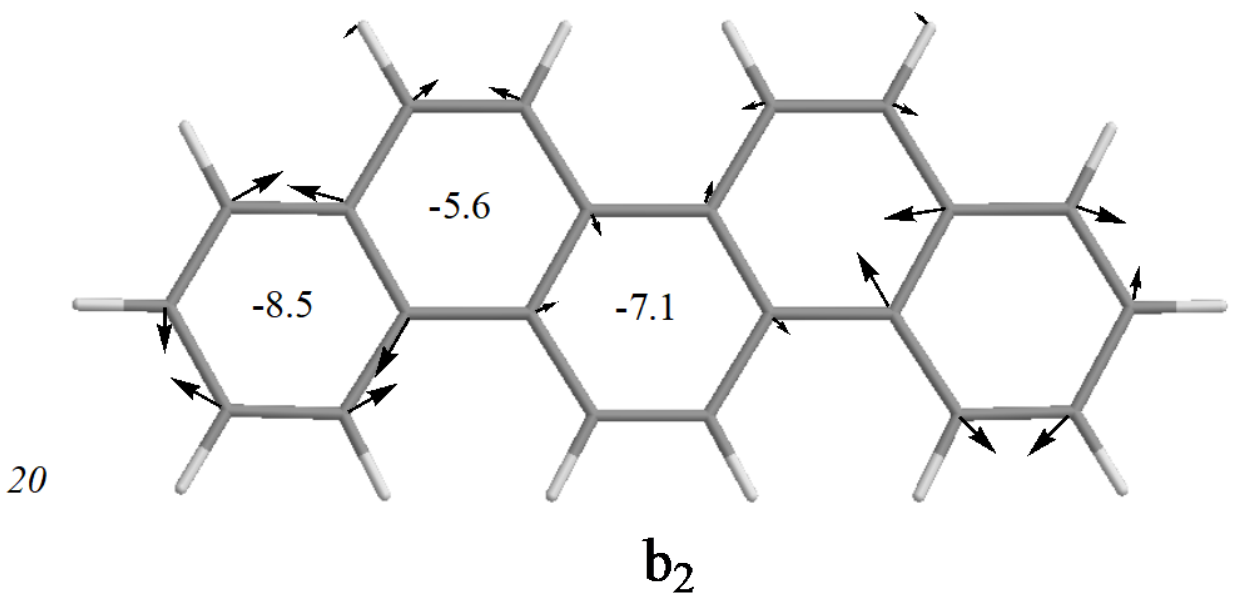

Figure 2c 


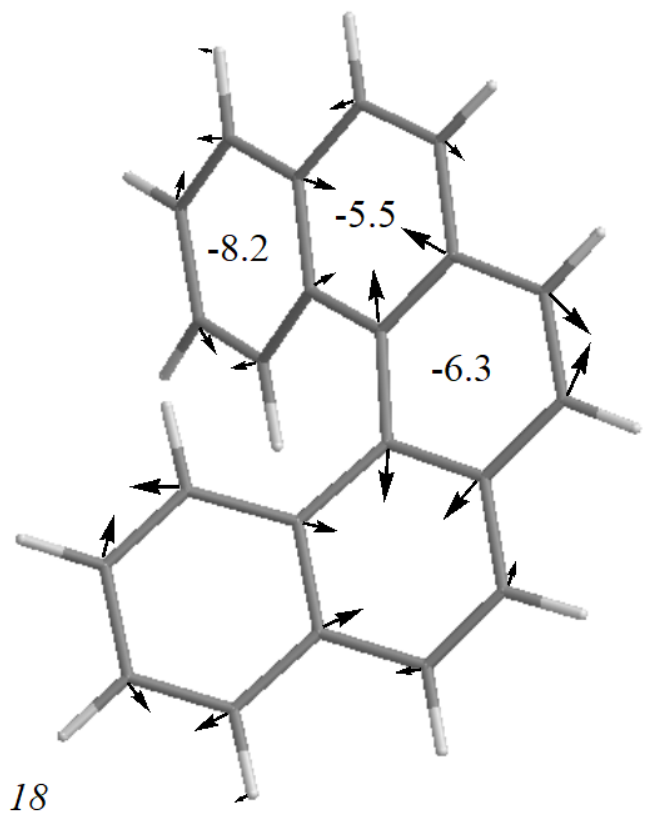

a (-2.211)

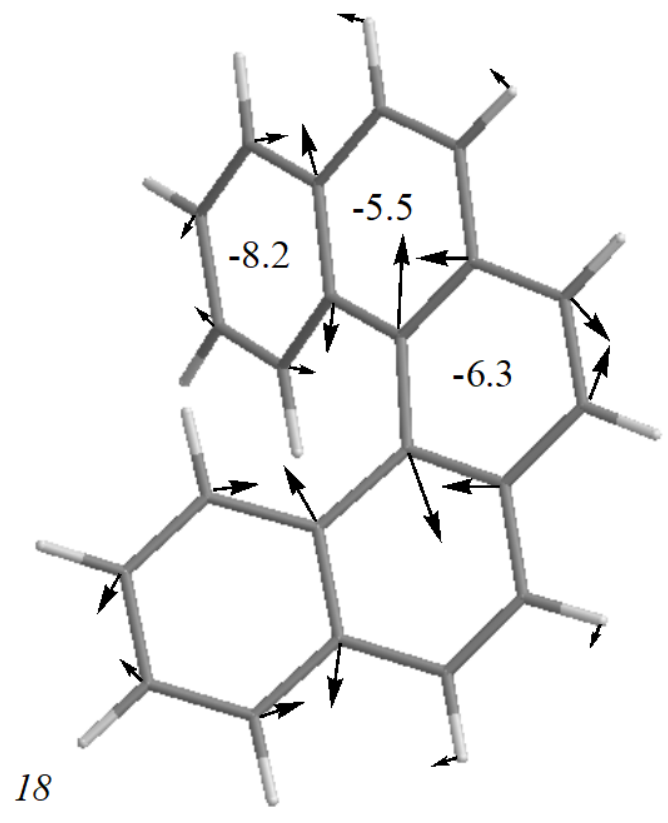

a (-1.114)
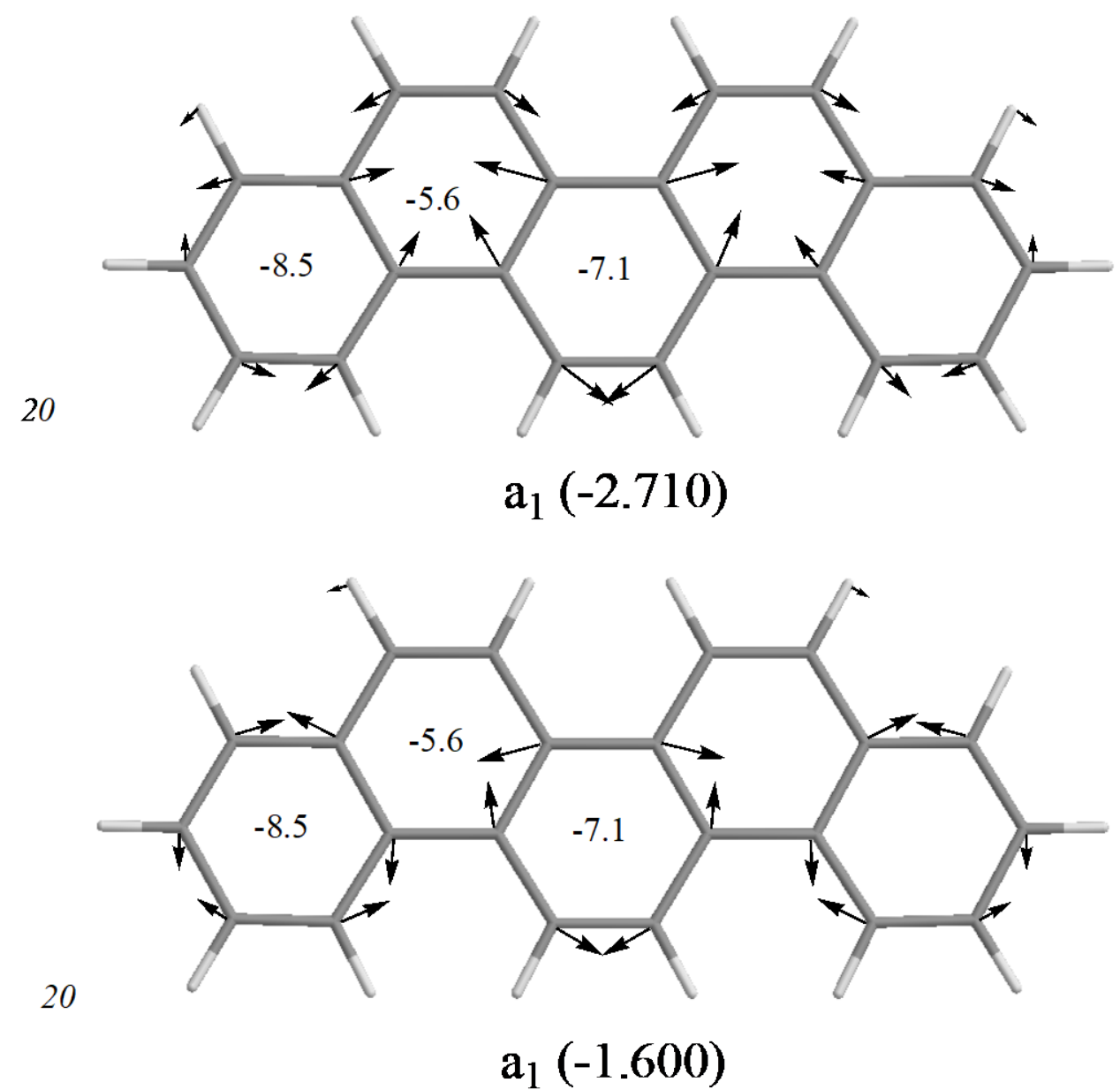

Figure 3 
Table 1: HF/6-31G, HF/6-311G(d), and B3LYP/6-311G(d) diagonal terms and eigenvalues of the Hessian matrices of the polarizability ( $\alpha$ ”) with respect to the nontotally symmetric modes, which break the GMPP for the smallest PAHs analyzed.

\begin{tabular}{|c|c|c|c|c|c|c|c|}
\hline \multirow{2}{*}{ molecules } & & \multicolumn{2}{|c|}{$\mathrm{HF} / 6-31 \mathrm{G}$} & \multicolumn{2}{|c|}{ HF/6-311G(d) } & \multicolumn{2}{|c|}{ B3LYP/6-311G(d) } \\
\hline & & diag. $\alpha ”$ & eigen. $\alpha ”$ & diag. $\alpha "$ & eigen. $\alpha "$ & diag. $\alpha "$ & eigen. $\alpha "$ \\
\hline \multirow[t]{2}{*}{ benzene } & $\mathrm{b}_{2 \mathrm{u}}$ & -0.425 & -1.077 & -0.914 & -1.356 & -0.506 & -0.872 \\
\hline & & -0.176 & & & & & \\
\hline \multirow[t]{2}{*}{ benzocyclobutadiene $^{\mathrm{a}}$} & $\mathrm{a}_{1}$ & -0.118 & -0.938 & -0.167 & -1.009 & -0.076 & -0.850 \\
\hline & & -0.091 & & -0.042 & & -0.037 & \\
\hline \multirow[t]{3}{*}{ naphthalene } & $\mathrm{b} 2 \mathrm{u}$ & -0.752 & -1.585 & -0.665 & -1.824 & -0.620 & -1.702 \\
\hline & & -0.174 & & -0.332 & & -0.207 & \\
\hline & & -0.032 & & -0.100 & & & \\
\hline \multirow[t]{3}{*}{ biphenylene } & $\mathrm{b}_{1 \mathrm{u}}$ & -0.680 & -2.368 & -0.983 & -3.030 & -1.402 & -2.356 \\
\hline & & -0.495 & & -0.922 & & -0.155 & -0.017 \\
\hline & & -0.387 & & -0.177 & & & \\
\hline \multirow[t]{2}{*}{ acenaphthylene } & $\mathrm{b}_{2}$ & -0.328 & -1.842 & -0.361 & -2.077 & & -1.601 \\
\hline & & -0.153 & & -0.309 & & & \\
\hline \multirow[t]{3}{*}{ pyracylene } & $\mathrm{b}_{2 \mathrm{u}}$ & -0.129 & -1.913 & -0.276 & -2.155 & -0.015 & -1.593 \\
\hline & & -0.105 & -0.035 & -0.224 & -0.083 & & \\
\hline & & -0.002 & & -0.050 & & & \\
\hline \multirow[t]{2}{*}{ fluorene } & b2 & -0.649 & -1.884 & -1.300 & -2.319 & -0.406 & -1.817 \\
\hline & & -0.184 & & & & -0.101 & -0.030 \\
\hline \multirow[t]{6}{*}{ anthracene } & $\mathrm{b}_{2 \mathrm{u}}$ & -2.015 & -3.905 & -1.819 & -4.502 & -1.825 & -3.527 \\
\hline & & -1.001 & -0.081 & -0.949 & -0.106 & -0.973 & -0.678 \\
\hline & & -0.069 & & -0.630 & & -0.462 & \\
\hline & & -0.022 & & -0.229 & & & \\
\hline & & & & -0.012 & & & \\
\hline & $\mathrm{b}_{1 \mathrm{u}}$ & & -0.013 & & -0.012 & & -0.002 \\
\hline
\end{tabular}

${ }^{\mathrm{a}}$ The diagonal terms and eigenvalues of the Hessian matrices of the polarizability ( $\alpha$ ”) are with respect to the totally symmetric modes. 
Table 2: HF/6-31G diagonal terms and eigenvalues of the hessian matrices of the polarizability ( $\alpha$ ") with respect to the nontotally symmetric modes, which break the GMPP for the heaviest PAHs analyzed.

\begin{tabular}{|c|c|c|c|c|c|c|c|}
\hline \multirow{2}{*}{ molecules } & & \multicolumn{2}{|c|}{ HF/6-31G } & \multirow{2}{*}{ molecules } & & \multicolumn{2}{|c|}{ HF/6-31G } \\
\hline & & diag. $\alpha "$ & eigen. $\alpha$ " & & & diag. $\alpha "$ & eigen. $\alpha$ " \\
\hline \multirow{2}{*}{$\begin{array}{l}\text { anthracene- } \\
\text { 9,10-dione }\end{array}$} & \multirow[t]{2}{*}{$\mathrm{b}_{2 \mathrm{u}}$} & -0.762 & -1.880 & perylene & b3g & -0.817 & -3.273 \\
\hline & & -0.384 & & & & -0.618 & -0.069 \\
\hline \multirow[t]{3}{*}{ acridine } & \multirow[t]{3}{*}{$\mathrm{b}_{2}$} & -2.359 & -3.930 & & & -0.452 & -0.055 \\
\hline & & -0.321 & -0.115 & & & -0.225 & \\
\hline & & -0.143 & & & $\mathrm{~b}_{2 \mathrm{u}}$ & -1.512 & -3.106 \\
\hline \multirow{5}{*}{$\begin{array}{c}\text { benzo[g] } \\
\text { quinoline }^{\mathrm{a}}\end{array}$} & \multirow[t]{5}{*}{$a^{\prime}$} & -1.250 & -3.798 & & & -0.276 & -0.018 \\
\hline & & -0.635 & -0.066 & & & -0.090 & \\
\hline & & -0.083 & -0.029 & & $\mathrm{~b}_{1 \mathrm{u}}$ & -0.004 & -0.169 \\
\hline & & -0.069 & -0.015 & & & & -0.104 \\
\hline & & -0.058 & & & $\mathrm{au}_{\mathrm{u}}$ & & -0.006 \\
\hline \multirow[t]{2}{*}{ phenanthrene } & \multirow[t]{2}{*}{$\mathrm{b}_{2}$} & -0.330 & -1.435 & triphenylene & $e^{\prime}$ & -0.921 & -1.797 \\
\hline & & -0.058 & & [5]helicene & b & -0.273 & -1.734 \\
\hline \multirow[t]{2}{*}{ [4]helicene } & \multirow[t]{2}{*}{$\mathrm{b}$} & -1.126 & -2.141 & & & -0.002 & -0.053 \\
\hline & & -0.122 & -0.360 & pentacene & b2u & -4.568 & -10.799 \\
\hline \multirow[t]{7}{*}{ tetracene } & \multirow[t]{5}{*}{$b_{2 u}$} & -3.832 & -6.812 & & & -2.662 & -1.526 \\
\hline & & -0.564 & -0.045 & & & -1.432 & -0.074 \\
\hline & & -0.454 & -0.028 & & & -1.036 & -0.015 \\
\hline & & -0.378 & & & & -1.014 & \\
\hline & & -0.326 & & & b3g & & -0.095 \\
\hline & $\mathrm{b}_{1 \mathrm{u}}$ & & -0.042 & & $\mathrm{~b}_{1 \mathrm{u}}$ & & -0.107 \\
\hline & b3g & & -0.045 & & & & -0.037 \\
\hline \multirow[t]{4}{*}{ chrysene } & \multirow[t]{4}{*}{$\mathrm{b}_{\mathrm{u}}$} & -0.916 & -2.321 & & $\mathrm{~b}_{3 \mathrm{u}}$ & & -0.014 \\
\hline & & -0.353 & -0.548 & picene & $\mathrm{b}_{2}$ & -0.138 & -2.131 \\
\hline & & -0.140 & & & & -0.017 & -0.214 \\
\hline & & -0.137 & & & & & \\
\hline
\end{tabular}

${ }^{\mathrm{a}}$ The diagonal terms and eigenvalues of the Hessian matrices of the polarizability ( $\boldsymbol{\alpha}$ ”) are with respect to the totally symmetric modes. 